\title{
Correction to: Positional cloning and comprehensive mutation analysis of a Japanese family with lithium-responsive bipolar disorder identifies a novel DOCK5 mutation
}

\author{
Hiromi Umehara - Masayuki Nakamura • Mio Nagai • Yuko Kato • Shu-ichi Ueno • Akira Sano
}

Published online: 19 October 2020

(c) The Author(s), under exclusive licence to The Japan Society of Human Genetics 2020

Correction to: Journal of Human Genetics

https://doi.org/10.1038/s10038-020-00840-7

The original version of this article unfortunately contained following mistakes.

"c.3150A $>\mathrm{G}$ " has to be changed to "c.3170A>G" in the following parts.

The line 11 in "Abstract".
The line 23 and 30 in "Linkage and haplotype analysis" in "Materials and methods".

The line 4 in "DNA sequence analysis using Sanger sequencing" in "Materials and methods".

The line 34 in "Discussion".

"g.25242432A $>$ G" has to be changed to "g.25224432A $>$ G" in the line 13 in "DNA sequence analysis" in "Results". 